\title{
Lipid Composition of Normal Male Rat Islets
}

\author{
Graciela B. Diaz", Ana Maria Cortizo, María Elisa Garcia and Juan José Gagliardino \\ Centro de Endocrinologia Experimental y Aplicada (UNLP_CONICET), Focultad de Ciencias Médicas, Universidad Nacional de La Plata, \\ Calles 60 y 120 (1900)-La Plata, Argentina
}

Lipid composition was studied in fresh isolated islets from normal male rats. Extractable lipids represent 1856 $\mu \mathrm{g}$ per $\mathrm{mg}$ islet protein. In such extracts, phospholipids and neutral lipids represent $13.5 \%$ and $86.5 \%$, respectively. Phosphatidylcholine $(\mathbf{4 5 . 8 \% )}$ and phosphatidylethanolamine $(20.6 \%)$ were the major components of the phospholipid fraction, and phosphatidylinositol $(8.9 \%)$ was the minor component. Esterified cholesterol (38.5\%), cholesterol $(25.5 \%)$ and free fatty acids $(24.4 \%)$ were the major components of the neutral lipid fraction. Fatty acids esterified to phospholipids account for $619.7 \mathrm{pmol} /$ islet, and $2710 \mathrm{pmol} / \mathrm{islet}$ were esterified to neutral lipids. In the phospholipid fraction, saturated and unsaturated fatty acids were in a similar proportion. Conversely, in the neutral lipids, two-thirds of the fatty acids were unsaturated. The $\omega 6$ family was the main component of the phospholipid unsaturated fatty acids. In the $\omega 6$ and $\omega 3$ families, the long-chain fatty acids represent the main components. In the neutral lipid fraction, a different percentage of each family was found: $\omega 3>\omega 6>\omega 9$. The long-chain polyunsaturated fatty acids were also predominant species in the $\omega 6$ and $\omega 3$ families. Further studies on the lipid composition of islets, obtained from rats with normal and altered islet functions, could provide new insights into the knowledge of the mechanism of insulin secretion.

Lipids 23, 1125-1128 (1988).

The ultrastructural and biochemical changes that occur in the pancreatic $\beta$-cell plasma membrane during emiocytosis, suggest that this organelle plays an active role in the last step of insulin secretion (1-3). However, several experimental evidences demonstrate that the cell membrane also participates in the earlier steps of this process, as a source of intracellular signals for the stimulus-secretion coupling (4). These signals would be released after the activation of several islet phospholipases (5). In this regard, the early release of arachidonic acid and its eicosanoid derivatives $(6,8)$, diacylglycerol (9), inositol-1,4,5-triphosphate $\left(\mathrm{IP}_{3}\right)(10)$ and other phosphoinositides components (11), as well as phosphatidic acid (12), were reported by several authors. Moreover, the increment in the total islet-phospholipid turnover in response to several insulin secretagogue agents has also been demonstrated $(4,13)$. All these data suggest that islet lipids, and particularly their phospholipid fractions, play an important role in the regulation of insulin secretion. Despite the large number of reports on this matter, little attention has been paid to the normal composition of islet lipids. This work was performed to provide such information through the quantitative determination of the

*To whom correspondence should be addressed.

Abbreviations: (F)FA, (free) fatty acid; $\mathrm{IP}_{3}$, inositol-1,4,5-triphosphate; NL, neutral lipid; PC, phosphatidylcholine; PE. phosphatidylethanolamine; PI, phosphatidylinositol; PL, phospholipid; PS, phosphatidylserine; SPH, sphingomyelin; DBI, double bond index; (HP)TLC, (high performance) thin layer chromatography; R, DBI/saturated FA ratio. different lipid fractions of fresh isolated islets from normal male rats.

\section{MATERIAL AND METHODS}

Adult normal male Wistar rats of ca. $200 \mathrm{~g}$ were fed ad libitum Purina Rat Chow (CABECA R N'3, Buenos Aires, Brazil). This chow contained $33 \%$ protein and $10 \%$ fat, and the following fatty acid composition: 16:0 (21.4\%), $16: 1(2.1 \%), 18: 0(8.2 \%), 18: 1(24.9 \%), 18: 2 \omega 6(37.7 \%)$ and $20: 4 \omega 6(0.2 \%)$. The animals had free access to drinking water. In the morning of the experiment, nonfasted rats were killed by cervical dislocation without anaesthesia and the tail of the pancreas was carefully dissected. Pancreatic islets were isolated from the pancreas by collagenase (Serva Feinbiochemica, Heidelberg, Germany) digestion (14). After such digestion, separation of the islets from the acinar tissue was achieved by several washings with cold buffer, sedimentation and handpicking under a stereomicroscope. Total lipids were extracted from groups of 150 islets with $19 \mathrm{ml}$ of a mixture of chloroform/methanol $(2: 1, \mathrm{v} / \mathrm{v})(15)$ and the washed lower phase was used for the analysis of the different lipid fractions. All solvents were of chromatographic grade. The total lipid content was determined by weighing the dry lipid extract resuspended in a small volume of chloroform. Neutral lipids (NL) were separated from phospholipids (PL) by thin layer chromatography (TLC) on plates of Silica Gel G-60, developed with chloroform/ methanol/acetic acid/water (90:6:1.0:0.75, v/v/v/v) (16). The spots were identified by iodine staining, scraped and the NL were eluted with chloroform/methanol $(2: 1, \mathrm{v} / \mathrm{v})$, and the Arvidson's method, chloroform/methanol/acetic acid/water (50:39:1:20, v/v/v/v) (17) was used for the elution of the PL. The extracts were evaporated to dryness under a stream of $\mathrm{N}_{2}$. Recovery control was performed at every step using an islet-lipid extract labeled with $\left[{ }^{14} \mathrm{C}\right]$ palmitic acid and appropriate standards labeled with $\left[{ }^{3} \mathrm{H}\right]$ glycerol. Final values were adjusted to the corresponding percentage recovery. A double control was used to assess the percentage composition of islet PL and neutral lipids, i.e., a scanner measurement performed directly on the thin layer plates and the determination of radioactivity in the samples eluted from those plates. Identical percentage values were obtained using either method.

Different fractions of NL or PL were separated on HPTLC plates (Silica Gel 60 F254 precoated for HPTLC, E. Merck, Darmstadt, Federal Republic of Germany). A developing solvent mixture consisting of petroleum ether/ diethyl ether/acetic acid (80:20:1, v/v/v) (18) was used to identify the different NL fractions. A solvent mixture of chloroform/methanol $/ 30 \%$ aq. ammonia/water (140:50:7:3, $\mathrm{v} / \mathrm{v} / \mathrm{v} / \mathrm{v})$ and of chloroform/methanol/acetic acid/water $(160: 20: 4: 1.5, \mathrm{v} / \mathrm{v} / \mathrm{v} / \mathrm{v})(19)$ was used to separate the PL fraction into its different constituents. Appropriate standards (P-L Biochemicals, Inc., Milwaukee, WI) were simultaneously run to identify each PL component. After chromatography, the lipids were charred for densitometry. 
Plates were dipped into a $3 \%$-cupric-acetate $(\mathrm{w} / \mathrm{v})-8 \%$ phosphoric-acid $(\mathrm{v} / \mathrm{v})$ solution and heated at $180^{\circ} \mathrm{C}$ for $15 \mathrm{~min}(20)$. The plates were later scanned using a Shimadzu scanning densitometer in the reflectance mode.

Fatty acid (FA) methyl esters from PL or NL were obtained by refluxing the extracts for $3 \mathrm{hr}$ at $65^{\circ} \mathrm{C}$ with $\mathrm{HCl} /$ methanol as described by Stöffel et al. (21). The methyl esters were then separated in a gas liquid chromatograph (GC-RIA Shimadzu) with a Supelco column (1-1851), $10 \%$ SP 2330 on 100/120 chromosorb AW Zog) and dual flame ionization detectors using a programmed temperature procedure.

The peaks obtained in the chromatograms were identified running appropriate standards of the purest available grade (P-L Biochemicals, Inc., Milwaukee, WI) and quantitatively estimated using the $\Delta 11-20: 1$ FA (P-L Biochemicals, Inc., Milwaukee, WI) as internal standard.

Protein concentration was determined according to Lowry's method (22), using bovine serum albumin (Sigma Chemical Co., St. Louis, MO) as standard.

Statistical analysis of the data was performed using the Student's t-test for independent samples.

\section{RESULTS}

Fresh isolated rat islets comprised $1856 \mu \mathrm{g}$ per $\mathrm{mg}$ of protein of total extractable lipids. Either the sum of the different islet-lipid fractions or the direct weight of the total islet-lipid extract gave similar figures for this parameter. From this total, the PL fraction represented $13.50 \%$, and the remaining $86.5 \%$ corresponded to NL. Table 1 shows the different species identified in the PL islet fraction. The largest percentage corresponded to phosphatidylcholine (PC) (45.8\%), followed by phosphatidylethanolamine (PE) $(20.6 \%)$, phosphatidylserine (PS) (14\%), sphingomyelin (SPH) (10.7\%) and phosphatidylinositol (PI) $(8.9 \%)$.

The different species identified in the islet-NL fraction are listed in Table 2. The major component was the esterified cholesterol $(38.5 \%)$ followed by cholesterol and free fatty acids (FFA) $(25.5 \%$ and $24.4 \%$, respectively), and the triacylglycerol fraction, the minor component.

The different FA identified in the islet-PL NL fractions are presented in Table 3 . The amount of FA esterified to PL was $619 \pm 50.8 \mathrm{pmol} /$ islet $(n=6)$, and $2710 \pm 188$ pmol/islet $(n=6)$ corresponds to the NL fraction.

\section{TABLE 1}

Phospholipid Composition of Fresh Isolated Islets

\begin{tabular}{lcccr}
\hline Phospholipids & ng/Islet & $\mu \mathrm{g} / \mathrm{mg}$ Protein & $\%$ \\
\hline Phosphatidylserine & $22.9 \pm$ & 1.4 & 35.2 & 14.0 \\
Phosphatidylinositol & $14.4 \pm 1.4$ & 22.2 & 8.9 \\
Sphingomyelin & $17.5 \pm 1.9$ & 26.9 & 10.7 \\
Phosphatidylcholine & $74.6 \pm 5.2$ & 114.8 & 45.8 \\
Phosphatidylethanolamine & $33.5 \pm$ & 4.1 & 51.5 & 20.6 \\
& & & & \\
Total & $162.9 \pm 11.10$ & 250.6 &
\end{tabular}

Phospholipid fractions were separated by HPTLC and the different species identified and quantitatively measured, as described in Material and Methods. Each value represents the mean \pm SEM of four different islet homogenates.
A similar percentage of saturated and unsaturated FA was found in the phospholipid fraction. Conversely, in the NL fraction, two-thirds of the FA were unsaturated. Accordingly, the double bond index (DBI) and the DBI/ saturated FA ratio (R) were larger in the NL than in the PL fraction.

The $\omega 6$ family represents the main component of the PL-unsaturated FA. In this family, the largest components were those with 20 and 22 carbons. A similar composition has been described in the outer acrosomal membranes of mammalian sperm (23). The long-chain polyunsaturated fatty acids also represent the main components of the $\omega 3$ family. A different percentage of each family was found in the NL fraction, namely $\omega 3>\omega 6>\omega 9$. As

\section{TABLE 2}

Neutral Lipid Composition of Fresh Isolated Islets

\begin{tabular}{lccc}
\hline Lipid & ng/Islet & $\mu g / m g$ Protein & $\%$ \\
\hline Cholesterol & $266 \pm 22$ & 409 & 25.5 \\
Free fatty acids & $254 \pm 10$ & 391 & 24.4 \\
Triacylglycerol & $122 \pm 12$ & 188 & 11.7 \\
Esterified cholesterol & $401 \pm 13$ & 618 & 38.5 \\
Total & $1043 \pm 29$ & 1606 & \\
\hline
\end{tabular}

Lipids were fractioned and quantitatively measured as described in the text. Each value represents the mean \pm SEM of four different islet homogenates.

\section{TABLE 3}

Fresh Islet Fatty Acids Composition

\begin{tabular}{lrr}
\hline Fatty acid & Phospholipis & Neutral lipids \\
\hline $12: 0$ & $0.8 \pm 0.2$ & $0.8 \pm 0.1$ \\
$14: 0$ & $3.3 \pm 0.5$ & $3.3 \pm 0.2$ \\
$15: 0$ & $1.5 \pm 0.3$ & $2.7 \pm 0.4$ \\
$16: 0$ & $22.5 \pm 2.6$ & $14.4 \pm 1.0$ \\
$16: 1$ & $6.1 \pm 0.9$ & $5.0 \pm 0.5$ \\
$18: 0$ & $15.1 \pm 1.7$ & $7.1 \pm 0.7$ \\
$18: 1 \omega 9$ & $13.3 \pm 1.3$ & $7.2 \pm 0.4$ \\
$20: 1 \omega 9$ & $0.2 \pm 0.0$ & $1.5 \pm 0.3$ \\
$20: 3 \omega 9$ & $0.3 \pm 0.1$ & $0.6 \pm 0.2$ \\
$18: 2 \omega 6$ & $4.2 \pm 0.4$ & $1.9 \pm 0.1$ \\
$18: 3 \omega 6$ & $0.7 \pm 0.3$ & $1.0 \pm 0.1$ \\
$20: 2 \omega 6$ & $0.1 \pm 0.0$ & $1.0 \pm 0.2$ \\
$20: 3 \omega 6$ & $0.7 \pm 0.3$ & $1.8 \pm 0.1$ \\
$20: 4 \omega 6$ & $4.6 \pm 0.6$ & $3.9 \pm 0.3$ \\
$22: 2 \omega 6$ & $5.6 \pm 1.1$ & $4.0 \pm 0.9$ \\
$22: 4 \omega 6$ & $4.5 \pm 0.7$ & $5.4 \pm 0.5$ \\
$22: 3 \omega 6$ & $3.2 \pm 0.7$ & $4.3 \pm 0.6$ \\
$18: 3 \omega 3$ & $1.3 \pm 0.2$ & $2.4 \pm 0.3$ \\
$20: 5 \omega 3$ & $0.3 \pm 0.2$ & $3.4 \pm 0.3$ \\
$22: 3 \omega 3$ & $4.8 \pm 0.9$ & $7.9 \pm 0.9$ \\
$22: 4 \omega 3$ & $3.2 \pm 0.7$ & $5.0 \pm 0.6$ \\
$22: 5 \omega 3$ & $2.9 \pm 1.1$ & $7.0 \pm 1.2$ \\
$22: 6 \omega 3$ & $0.7 \pm 0.5$ & $8.4 \pm 1.2$ \\
Sat FA & 43.3 & 28.5 \\
DBI & 141.7 & 240.8 \\
R & 3.3 & 8.5 \\
\hline
\end{tabular}

The lipids were interesterified and the resulting fatty acid methyl esters were analyzed as described in the text. Each value represents the mean percentage \pm SEM of four determinations; DBI $=$ double bond index; $\mathrm{R}=\mathrm{DBI} /$ saturated $\mathrm{FA}$ ratio. 
in the PL fraction, long-chain polyunsaturated FA were the predominant species found in the $\omega 6$ and $\omega 3$ families.

\section{DISCUSSION}

It is well known that the lipid composition of animal tissues, even among a given species, varies from one tissue to another, being affected by several factors such as diet composition, sex, age, circadian rhythms and environmental conditions (24). In our experimental model, the effect of the enzymes from the exocrine pancreas released during the isolation of islets, could also represent another source of variation. In islets isolated from normal nonfasted male Wistar rats, such composition shows an uneven proportion of polar $(13.5 \%)$ and neutral lipids $(86.5 \%)$.

The total content of PL currently described, expressed on a per-islet basis, agrees with the values reported by Hallberg (25) in Wistar rats. Conversely, they are significantly lower than the values reported by Montague and Parkin (26) in guinea pig islets. According to Hallberg (25), this difference probably reflects species variations of the total PL content of islets. Hallberg stated also that the total content of PL is not affected by different conditions like 48-hr starvation, the culture of the isolated islets for one week, the addition of leucine and gliclazide to or the removal of $\mathrm{Ca}^{2+}$ from such medium (25). We have previously shown that the PL content was significantly lower in islets isolated from hypothyroid rats (27). Hence, those results might indicate that chronic rather than acute conditions (or effects) can modify the total content of islet PL, which could affect the release of insulin.

PL fractions identified in the islets are similar to those described in other rat tissues. PC and PE are their major components, as in normal liver $(24,28)$ with PS instead of PI being the minor component in the islets. Otherwise, the percentages of PC and PE are lower in the islets, but PS and SPH are higher than in the liver. The relative content of the different PL classes currently described in the islets isolated from normal nonfasted Wistar rats, with $\mathrm{PC}$ and $\mathrm{PE}$ as major components, coincides with the data reported from obese hyperglycemic (29) or NMRI mice (25) and Sprague-Dawley rats (30). This islet PL composition is significantly altered in some pathological conditions such as hypothyroidism (27), which is accompanied by a decrease in the insulin secretory response to glucose. These data suggest that the normal islet-PL composition might be a condition necessary to achieving an adequate $\beta$-cell secretory response.

The amount of NL per mg of protein was similar in liver and isolated islets (31). However, the esterified cholesterol, the cholesterol and the FFA fractions were larger, but the triacylglycerol fraction was lower in the islets compared to liver.

The islets, like other rat tissues, present a complex composition of esterified FA, with a clear predominance of even-numbered acids (32). Berne has concluded that the major source for these FA is their extracellular uptake rather than their biosynthesis (33). Palmitic acid was the largest component of the saturated FA as in the heart (34). This fact could, at least in part, explain: a) the high and concentration-dependent oxidation rate of palmitic acid measured in islets (35) and b) the large incorpora- tion of labeled palmitate into the islet lipids by islets incubated in the presence of different insulin secretagogue agents (36).

Turk et al. have already reported on the FA composition of the major glycerolipids from Sprague-Dawley-rat islets cultured overnight and then incubated with glucose (30). No references are given in this paper on the FA composition in freshly untreated islets. As shown in our Table 3, they also found that palmitate and stearate were the predominant saturated FA esterified to PL. Conversely, some discrepancies were found in the composition of unsaturated FA. We have previously reported that the percentage composition of polyunsaturated FA changed significantly in islets incubated with high glucose (37). This effect was attributed to a possible effect of glucose on the activity of islet desaturases and the FA chain-elongation process (37). Hence, the different metabolic conditions of the islets used in the current report and those mentioned above could, at least in part, explain the apparent discrepancies in the percentage distribution of polyunsaturated FA. The different number of FA considered in Turk's study compared with our own (11 vs 22), could also contribute to such a difference. However, we do not have a plausible explanation for the particular lower percentage of arachidonic acid found in our experiments.

A large number of a long-chain polyunsaturated FA was found in the islets, mainly esterified to the neutral lipids. The richness of polyunsaturated FA is a characteristic of the lipid composition found in very active tissues, such as heart and liver (38). Such composition explains the large DBI and R (DBI/saturated acid ratio) found in the islets (37). The degree of unsaturation of the phospholipid acyl chains has been considered one of the main determinants of the microviscosity of lipid regions (39). Assuming that the PL content grossly represents the islet membrane composition, the values obtained for such a parameter would suggest that, in the islets, the microviscosity of the membrane lipids is lower than in the liver membranes.

It has already been reported that the oxidation of endogenous FA accounts for about $30 \%$ of islet basal respiratory rate (40). The large amount of endogenous islet FA, mainly esterified to the neutral lipid fraction, could easily provide such an oxidizable substrate. In addition, the FA also represent the source of intracellular signals for the stimulus-secretion coupling. The change in the mass of FA esterified to PC (30), the release of arachidonic acid $(6,7)$ and its eicosanoid derivatives $(6-8,36)$ under the glucose stimulus are clear examples of such function.

Further, more detailed studies on the composition and metabolism of islet lipids in normal and pathological conditions would provide important evidence for a more comprehensive knowledge of the insulin secretion mechanism.

\section{ACKNOWLEDGMENTS}

This paper was partially supported by funds from CONICET, CICPBA and Ministerio de Salud de la Provincia de Buenos Aires. The authors are greatly indebted to INIBIOLP and CIDCA authorities for providing technical support and the use of equipment. G.B.D. is a Fellow from CICPBA; A.M.C. is a Member of the Carrera del Investigador CICPBA; M.E.G. is a Member of the Carrera del Personal de Apoyo Profesional CICPBA; and J.J.G. is a Member of the Carrera del Investigador CONICET. 


\section{REFERENCES}

1. Orci, L. (1982) Diabetes 31, 538-565.

2. Lacy, P.E. (1961) Am. J. Med. 31, 851-859.

3. Semino, M.C., Gagliardino, E.P., and Gagliardino, J.J. (1987) Acta Physiol. Pharmacol. Latinoam. 37, 533-539.

4. Best, L., and Malaisse, W.J. (1983) Diabetologia 25, 299-305.

5. Kelly, K.L., and Laychock, S.G. (1981) Prostaglandins 21, 759-769.

6. Gagliardino, J.J., Borelli, M.I., Gagliardino, E.P., and García, M.E. (1986) Diabetes Res. Clin. Practice 1, 327-333.

7. Wolf, B.A., Turk, J., Sherman, W.R., and McDaniel, M.L. (1986) J. Biol. Chem. 261, 3501-3509.

8. Robertson, R.P. (1988) Diabetes 37, 367-370.

9. Tanigawa, L., Kuzuya, H., Imura, H., Taniguchi, H., Baba, S., Takai, Y., and Nishizyku, Y. (1982) FEBS Lett. 138, 183-186.

10. Prentki, M., Biden, T.J., Janjic, D., Irvine, R.F., Berridge, M.J. and Wollheim, C.B. (1984) Nature 309, 562-564.

11. Zawalich, W.S. (1988) Diabetes 37, 137-141.

12. Dunlop, M.E., Larkins, R.G., and Court, J.M. (1982) FEBS Lett. 144, 259-263.

13. Best, L., Dunlop, M., and Malaisse, W.J. (1984) Experientia 40, 1085-1091.

14. Lacy, P.E., and Kostianovsky, M. (1967) Diabetes 16, 35-39.

15. Folch, J., Lee, S.M., and Sloan-Stanley, G.M. (1957) J. Biol. Chem. 226, 497-509.

16. Van Dorp, D.A., Beerthuis, R.K., Nugteren, D.W., and Vankerhan, H. Jr. (1964) Biochim. Biophys. Acta 10, 204-206.

17. Arvidson, G.A.E. (1968) Eur. J. Biochem. 4, 478-486.

18. Malins, D., and Mangold, H.K. (1960) J. Am. Oil Chem. Soc. 37, 576-578.

19. Neskovic, N.M., and Kostic, D.M. (1968) J. Chromatogr. 35, 297-300.

20. Macala, L.J., Yu, R.K., and Ando, S. (1983) J. Lipid, Res. 24, 1243-1250.

21. Stöffel, O.W., Chu. F., and Ahrens, E.W., Jr. (1959) Anal Chem.
$31,307-308$

22. Lowry, O.W., Rosebrough, N.J., Farr, A.L., and Randall, R.H. (1951) J. Biol. Chem. 193, 265-275.

23. Nikolopoulou, M., Soucek, D.A., and Vary, J.C. (1986) Lipids $21,566-570$.

24. Ruggieri, S., and Fallani, A. (1979) Lipids 14, 323-333.

25. Hallberg, A. (1984) Biochim. Biophys. Acta 796, 328-335.

26. Montague, W., and Parkin, E.N. (1980) Horm. Metab. Res. (Suppl) 10, 153-157.

27. Cortizo, A.M., García, M.E., Pasquini, J.M., and Gagliardino, J.J. (1984) IRCS Med. Sci. 12, 1117-1118.

28. Upreti, G.C., de Antueno, R.J., and Wood, R. (1983) J. Natl. Cancer Inst. 70, 567-573.

29. Fex, G., and Lernmarck, A. (1972) FEBS Lett. 25, 287-291.

30. Turk, J., Wolf, B.A., Lefkowith, J.B., Stump, W.T., and McDaniel, M.L. (1986) Biochim. Biophys. Acta 879, 399-409.

31. Upreti, G.C., de Antueno, R.J., and Wood, R. (1983) J. Nath Cancer Inst. 70, 559-566.

32. Brenner, R.R., Mercuri, O., and De Tomas, M.E. (1962) J. Nutr. 77, 203-209.

33. Berne, C. (1975) Biochem. J. 152, 667-673.

34. Peluffo, R.O., and Brenner, R.R. (1962) An. Asoc. Quím. Argent. 50, 1-12.

35. Berne, C. (1975) Biochem. J. 152, 661-666.

36. Laychock, S.G. (1983) Diabetes 32, 6-13.

37. Cortizo, A.M., Garcia, M.E., and Gagliardino, J.J. (1987) Med. Sci. Res. 15, 289-290.

38. Di Corleto, P.E., Warrach, J.B., and Silversmith, D.B. (1979) J. Biol. Chem. 254, 7795-7802.

39. Shinitzky, M., and Inbar, M. (1976) Biochim. Biophys. Acta 433 , 133-149.

40. Malaisse, W.J., Malaisse-Lagae, F., Sener, A., and Hellerstrom, C. (1985) Biochem. J. 227, 995-1002.

[Received August 21, 1987; Revision accepted July 5, 1988] 